\title{
Lattice Boltzmann simulation of permeability of cement-based materials
}

\author{
M. Zhang \& G. Ye \\ Microlab, Faculty of Civil Engineering and Geosciences
}

Delft University of Technology, Delft, The Netherlands

\begin{abstract}
Due to its importance on the durability assessment and service life prediction of reinforced concrete structures, it is essential to estimate the permeability of cement-based materials. This paper presents a microstructure-based permeability modeling of cement-based materials using the lattice Boltzmann (LB) method, where a multiple-relaxation-time (MRT) corrosion operator is utilized. The MRT-LB model is applied to simulate the single-phase fluid flow in saturated cement paste. The microstructure of cement paste is generated by HYMOSTRUC3D. Following the onset of steady-state flow, the overall mean velocity is obtained and the water permeability is calculated according to Darcy's law. In the numerical experiments, the effects of water-to-cement (w/c) ratio, degree of hydration and effective porosity on the water permeability of cement paste are evaluated. The simulations are validated with experimental data obtained from the literature. The results indicate that the simulated permeability is close to the measured permeability for the samples at early age. For the samples with effective porosity higher than $20 \%$, the simulation shows a good agreement with the experiment. In addition, for both simulation and experiments, the relationship between the water permeability and the effective porosity is highly correlated. This implies that the effective porosity is a critical parameter which determines the permeability of cement-based materials.
\end{abstract}

\section{INTRODUCTION}

It is well known that the permeability of a cementbased material plays a crucial role in durability assessment of concrete structure, since the durability of the cement-based material is strongly dependent on the possibility of penetration of aggressive agents into the porous material with water as medium. In view of its importance, a number of models have been proposed and developed in the past to estimate the permeability of cement-based materials. Critical reviews on these models were given by Marchand and Gerard (1997) and Ye (2003). In principle, an ideal permeability model should be based on direct measurements of the microstructure, especially the pore structure of a representative sample of the material because the permeability strongly depends on the microstructure of the material (Ye 2003). In order to characterize the microstructure, different techniques including experimental tests and numerical simulation are proposed and developed in the past decades. With respect to numerical simulation, CEMHYD3D (Bentz 1997) and HYMOSTRUC3D (van Breugel 1991) are widely used.

In recent years, significant progress has been made in the microstructure-based modeling of fluid flow in porous media. One of the most promising methods is the lattice Boltzmann (LB) method. The LB approach simulates flow phenomena through tracking particles that move and collide in space under rules that the collision does not result in mass and momentum changes. Because of its nature, the LB method has several advantages such as intrinsic parallelism, ease of implementation and ability to handle complex boundary conditions (Pan et al. 2004). In addition, the LB method provides insight into the internal velocity distribution and allows one to detect preferential flow paths in the complex microstructure. Therefore, the LB method seems very suitable for simulating fluid flow in the porous material with a complex microstructure, e.g. cementbased materials, and offers a better understanding of fluid flow processes in the porous material.

The purpose of this paper is to predict the permeability of cement-based materials via the lattice Boltzmann method. As an example, cement paste is considered. Three-dimensional microstructure of cement paste is generated by HYMOSTRUC3D. The multiple-relaxation-time lattice Boltzmann (MRTLB) model is utilized to simulate the single-phase fluid flow in the obtained microstructure of cement paste. The fluid flow simulation is run until steadystate flow condition is achieved. The mean velocity of steady-state flow in the entire specimen in the 
flow direction is obtained and used as input for permeability calculation according to Darcy's law. The results of simulated water permeability are verified with experimental data obtained from the literature.

\section{MULTIPLE-RELAXATION-TIME LATTICE BOLTZMANN (MRT-LB) SIMULATION}

\subsection{Lattice Boltzmann method}

At the microscopic level, a fluid made up of particles (the fluid molecules) can move randomly in all directions as a result of collisions of particles with other particles. The probability of these particles' position in the physical space is dynamically described by the continuous Boltzmann equation. The LB method approximates the continuous Boltzmann equation by discretizing the physical space with regular lattice nodes and setting the velocity space as microscopic velocity vectors. The microscopic movements of particles at each lattice node are represented by the particle distribution functions. The evolution of particle distribution functions satisfies the following discrete lattice Boltzmann equation (Qian et al. 1992):

$$
f_{i}(x+\delta x, t+\delta t)=f_{i}(x, t)+\Omega_{i}
$$

where $f_{i}$ is the non-equilibrium particle distribution function at location $x$ at time $t, \Omega_{i}$ stands for the collision operator. $\tau$ is the relaxation time which is related to the kinematic viscosity of fluid $(v)$ as $v=c_{s}^{2} \cdot(\tau-0.5)$ with sound speed $c_{s}^{2}=1 / 3 \cdot(\delta x / \delta t)$, herein, the lattice-spacing $\delta x$ and time-step $\delta t$ are defined as $1 \mathrm{lu}$ and $1 \mathrm{ts}$ (lu and ts refer to lattice units and time step, respectively in the LB method). The subscript $i$ represents the number of allowed velocity directions on the lattice. In this study, we use a very popular D3Q19 model (as shown in Figure 1), which is a three-dimensional model with nineteen microscopic velocity vectors.

For the D3Q19 model, the equilibrium distribution function $f_{i}^{e q}$ is given by

$$
f_{i}^{e q}=w_{i} \rho\left[1+\frac{\boldsymbol{e}_{i} \cdot \boldsymbol{u}}{c_{s}^{2}}+\frac{\left(\boldsymbol{e}_{i} \cdot \boldsymbol{u}\right)^{2}}{2 c_{s}^{4}}-\frac{\boldsymbol{u} \cdot \boldsymbol{u}}{2 c_{s}^{2}}\right]
$$

where $\rho$ and $\boldsymbol{u}$ are the macroscopic density and velocity respectively, $w_{i}$ is the weight factor associated with $i^{\text {th }}$ direction which is given as $w_{i}=1 / 3(i=0)$, $w_{i}=1 / 18(i=1,2, \ldots, 6)$ and $w_{i}=1 / 36(i=7,8, \ldots$ ,18). The macroscopic local density (mass) $\rho(x)$ and velocity $\boldsymbol{u}(x)$ of each lattice node are related to the distribution function by

$$
\rho=\sum_{i=0}^{n} f_{i}, \quad \boldsymbol{u}=\frac{1}{\rho} \sum_{i=0}^{n}\left(f_{i} \cdot e_{i}\right)
$$

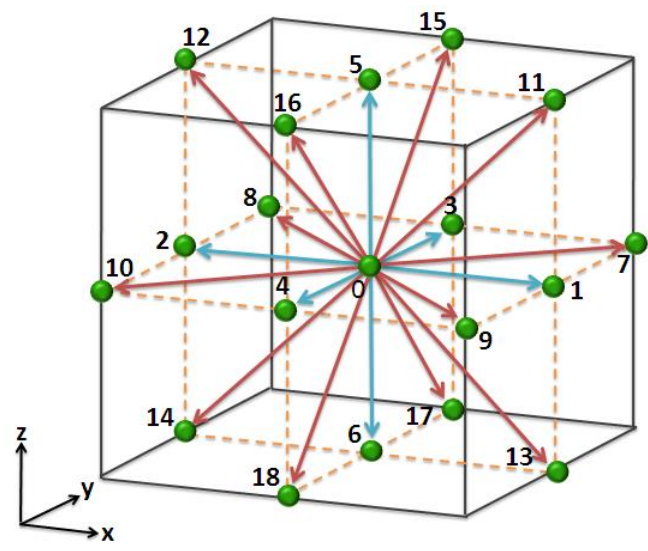

Figure 1: Lattice velocity direction of the D3Q19 model.

\subsection{Multiple-relaxation-time model}

A multiple-relaxation-time (MRT) model proposed by d'Humières et al. (2002) is used in this study, which separates the kinetic modes from the relaxation times and allows tuning to improve the numerical stability and eliminate the viscosity dependent characteristic. The collision operator of MRT model is given by:

$\Omega_{i}=-M^{-1} \hat{S}\left[m_{i}(x, t)-m_{i}^{e q}(x, t)\right]$

where the collision matrix $\hat{S}=M \cdot S \cdot M^{-1}$ is a diagonal relaxation matrix, the transformation matrix $M$ relates the distribution functions $f$ and $f^{e q}$ to their moments $m$ and $m^{\mathrm{eq}}$ as in the following:

$m=M \cdot f, \quad m^{e q}=M \cdot f^{e q}$

The transformation matrix $M$ can be constructed from the monomials of Cartesian components of the discrete velocities by applying the Gram-Schmidt orthogonalization procedure (Lallemand \& Luo 2003). The row vectors of $M$ are mutually orthogonal, i.e., $M^{-1} M^{\mathrm{T}}$ is a diagonal matrix, but not normalized, so that its matrix elements are integers. For the D3Q19 model, the 19 moments are arranged in the following order (d'Humières et al. 2002):

$$
\begin{aligned}
m= & \left(m_{0}, m_{1}, \cdots, m_{18}\right)^{T}=\left(\rho, e, \varepsilon, j_{x}, q_{x}, j_{y}, q_{y}, j_{z},\right. \\
& \left.q_{z}, 3 p_{x x}, 3 \pi_{x x}, p_{w w}, \pi_{w w}, p_{x y}, p_{y z}, p_{x z}, m_{x}, m_{y}, m_{z}\right)^{T}
\end{aligned}
$$

where $\rho$ is the density mode, $e$ is the energy mode, $\varepsilon$ is related to energy square, $j_{x}, j_{y}$ and $j_{z}$ represent the $x, y$ and $z$ components of momentum (mass flux), $q_{x}$, $q_{y}$ and $q_{z}$ correspond to the $x, y$ and $z$ components of energy flux, $p_{x x}, p_{w w}, p_{x y}, p_{y z}, p_{x z}, \pi_{x x}$ and $\pi_{w w}$ are related to the symmetric traceless viscous stress tensor.

The diagonal relaxation matrix $\hat{S}$ composed of a series of relaxation rates is given by: 


$$
\begin{aligned}
& \hat{S}=\operatorname{diag}\left(0, s_{e}, s_{\varepsilon}, 0, s_{q}, 0, s_{q}, 0, s_{q},\right. \\
& \left.\qquad s_{v}, s_{\pi}, s_{v}, s_{\pi}, s_{v}, s_{v}, s_{v}, s_{m}, s_{m}, s_{m}\right)
\end{aligned}
$$

in which the relaxation rate $s_{v}$ is related to the kinematic viscosity of the fluid as:

$s_{v}=\frac{2}{6 v+1}=\frac{1}{\tau}$

The remaining relaxation rates, i.e., $s_{e}, s_{\varepsilon}, s_{q}, s_{\pi}$ and $s_{m}$ can be freely chosen within the range from 0 to 2 and can be adjusted to improve the accuracy as well as the stability of the MRT model. The optimal values of them depend on the specific problem under consideration, such as the geometry, initial conditions and boundary conditions. It was found that a good choice of these relaxation rates for stokes flow is (d'Humières et al. 2002):

$s_{e}=s_{\varepsilon}=s_{\pi}=s_{v}, \quad s_{q}=s_{m}=8 \frac{2-s_{v}}{8-s_{v}}$

Implementation of the MRT-LB model consists of two main steps: a collision step and a streaming step. The collision step is to calculate the distribution function components, that is, the right-hand side term on equation (1) as $f_{i}^{*}(x, t)=f_{i}(x, t)+\Omega_{i}$ and the streaming step is to propagate $f_{i}^{*}(x, t)$ to the neighboring nodes and generate the non-equilibrium distribution function for the next time step as $f_{i}(x+\delta x, t+\delta t)=f_{i}^{*}(x, t)$. After this, the density and velocity at each lattice node are updated using equation (3).

\subsection{Units conversation}

Fluid transport in cement-based materials involves three basic physical units, i.e. length, time and mass, which are distinct from the corresponding lattice units used in the LB simulations. For the practical application of LB simulation, it is essential to establish a mapping between the lattice units and real physical units: $x L_{0} \rightarrow x_{p}$ (length), $t T_{0} \rightarrow t_{p}$ (time) and $\rho M_{0} \rightarrow \rho_{p}$ (mass). With these dimensionless variables $(x, t, \rho)$, the simulation result can be related to any quantities in the physical system, for example, velocity from lattice-spacing per time-step (lu/ts) in the simulation matches to meter per second $(\mathrm{m} / \mathrm{s})$ in the physical system.

The length mapping $L_{0}$ is determined from the chosen resolution in the simulation. For example, in the modeling of permeability of cement paste, the representative elementary volume (REV) of cement paste is $100 \times 100 \times 100 \mu^{3}$. It is digitized into $200 \times 200 \times 200$ lattices. Thus, the resolution $\delta x_{p}=0.5$ $\mu \mathrm{m}=5 \times 10^{-7} \mathrm{~m}$. Since a lattice spacing $\delta x=1$, hence, $L_{0}=\delta x_{p} / \delta x=5 \times 10^{-7} \mathrm{~m}$.

The mass mapping is deduced from the fluid density. For example, the density of water at $20{ }^{\circ} \mathrm{C}$ is given as $\rho_{w, p}=1000 \mathrm{~kg} / \mathrm{m}^{3}$. In the simulation, the density at all lattice nodes is set to $\rho=1$. Since density has dimensions $[\mathrm{M}][\mathrm{L}]^{-3}, \rho_{w, p}=M_{0} L_{0}^{-3}$, hence, $M_{0}=1.25 \times 10^{-16} \mathrm{~kg}$.

The time mapping $T_{0}$ can be obtained from the user's choice of fluid kinematic viscosity $v_{w, p}$. For water at $20^{\circ} \mathrm{C}, v_{w, p}=1.004 \times 10^{-6} \mathrm{~m}^{2} / \mathrm{s}$. It has dimensions $[\mathrm{L}]^{2}[\mathrm{~T}]^{-1}$. From the dimensions of fluid kinematic viscosity, it can be deduced that $v_{w, p}=v L_{0}^{2}$. In the LB model, the lattice viscosity $v$ is related to the relaxation time $\tau$ and the sound speed $c_{s}$ according to $v=c_{s}^{2}(\tau-0.5)$. Suppose that $\tau=1$, we can get $v=$ $1 / 6$, hence, $T_{0}=4.15 \times 10^{-8} \mathrm{~s}$.

\subsection{Permeability calculation}

In the LB simulation, a density (pressure) difference is applied at the inlet and outlet sides (in the $z$ direction) to drive the flow. The velocity and unknown particle distribution can be calculated according to pressure boundary proposed by Zou and He (1997). Other four sides are considered as periodic boundaries, so that the nodes of opposite boundaries are treated as neighboring. Thus, the particle distributions moving out the domain from one side will re-enter the domain through its opposite side during the streaming step. The half-way bounce-back (Zhang et al. 2011) is imposed on the pore-solid interface, thus, the distribution functions from the pore node streaming to their neighboring solid nodes scatter back to the pore node along its incoming direction. The LB simulation is run until the steady-state flow condition is reached. The steady-state flow criterion is defined such that the difference in the average velocity of the whole domain between two consecutive time steps is less than a specified threshold value. This threshold value is selected to be $1.0 \times 10^{-}$ 7, which was found not only sufficient to guarantee the computational accuracy but also helpful to reduce the computing time and memory requirements. Once the steady-state flow condition is satisfied, the permeability can be calculated according to Darcy's law and expressed in terms of LB parameters:

$$
\kappa_{l b}=\frac{v<u_{z}>}{\nabla p}=v \cdot \frac{N_{z} \cdot \delta x}{c_{s}^{2} \cdot \Delta \rho} \cdot \frac{1}{N_{x} \cdot N_{y} \cdot N_{z} \cdot \delta x^{3}} \sum u_{z}
$$

where $\kappa_{l b}$ represents the intrinsic permeability in LB units, $v$ is the kinematic viscosity of fluid, $\left\langle u_{z}\right\rangle$ denotes the overall mean value of velocity in the $z$ direction, $\nabla p$ is the pressure gradient, $\nabla \rho$ stands for the density difference between the inlet and outlet, $N_{x}, N_{y}$ and $N_{z}$ are the numbers of discretization nodes in the $x, y$ and $z$-direction, respectively.

In the physical system, the intrinsic permeability of a porous material $(\kappa)$ has dimensions of length squared. On the basis of units conversion as described above, the intrinsic permeability in physical units can be obtained from that in LB units, i.e. $\kappa_{l b}$, according to the relation $\kappa=\kappa_{l b} L_{0}^{2}$. If the fluid in- 
volved is water, the intrinsic permeability can be converted into saturated water permeability $k_{w}(\mathrm{~m} / \mathrm{s})$ by multiplying by a conversion coefficient $\rho_{\mathrm{p}} g / \eta_{\mathrm{p}}\left(\mathrm{m}^{-}\right.$ $\left.{ }^{1} \mathrm{~s}^{-1}\right)$ in which $\rho_{\mathrm{p}}$ and $g$ represent the fluid density $\left(\mathrm{kg} / \mathrm{m}^{3}\right)$ and acceleration of gravity $\left(9.81 \mathrm{~m} / \mathrm{s}^{2}\right) \mathrm{re}-$ spectively. For water at a temperature of $20^{\circ} \mathrm{C}$, this conversion coefficient equals $9.76 \times 10^{6} \mathrm{~m}^{-1} \mathrm{~s}^{-1}$.

\section{3D MICROSTRUCTURE OF PORTLAND CEMENT PASTE}

As an example, the LB simulation of permeability of Portland cement paste is demonstrated herein. The 3D microstructure of Portland cement paste used in the simulation is generated by the HYMOSTRUC3D model (van Breugel 1991, Ye 2003). The HYMOSTRUC3D is designed to simulate the hydration process and microstructure evolution of cementbased materials. In the model, the hydration of microstructure is simulated as a function of the particle size distribution (PSD), the chemical composition of the cement, the water-to-cement (w/c) ratio and the reaction temperature. The simulated $3 \mathrm{D}$ microstructure of hydrating cement paste is modeled as a composite structure consisting of pores, un-hydrated cement grains and hydration products, i.e. inner and outer hydration products.

The simulated microstructure is subsequently digitized into a three-dimensional array of voxels with an identical resolution (side length). The resolution in the digitized microstructure might affect the simulation accuracy. In general, the higher the resolution is, the more accurate the solution is, especially when the porosity of samples is low. However, using higher resolution would significantly increase the demand on computer resources and computational time. In order to select a suitable and reasonable resolution, the influence of the resolution on the ionic diffusivity in saturated hardened cement paste was estimated and presented in Zhang et al. (2011). It was indicated that the resolution of $0.5 \mu \mathrm{m} / \mathrm{voxel}$ was not only suitable for the solution accuracy but also satisfied the computational efficiency. Therefore, resolution of $0.5 \mu \mathrm{m} / \mathrm{voxel}$ is chosen in the following simulations. The voxels are assigned to be capillary pore or un-hydrated cement or hydration product, depending on their corresponding positions in the system. An example of the digitized microstructure with size of $100 \times 100 \times 100 \mu \mathrm{m}$ and the extracted pore structure of cement paste with w/c 0.5 at the age of 26 days is shown in Figure 2. The cement used in the simulation was Portland cement CEM I $42.5 \mathrm{~N}$, the main constituents of which are $\mathrm{C}_{3} \mathrm{~S}=$ $63.53 \%, \mathrm{C}_{2} \mathrm{~S}=13 \%, \mathrm{C}_{3} \mathrm{~A}=8.43 \%, \mathrm{C}_{4} \mathrm{AF}=9 \%$, respectively. The Blaine fineness of the cement was $420 \mathrm{~m}^{2} / \mathrm{kg}$. A continuous particle size distribution (PSD) with the minimum size of $1 \mu \mathrm{m}$ and the max- imum size of $50 \mu \mathrm{m}$ was used. The curing temperature was $20^{\circ} \mathrm{C}$.

(a)

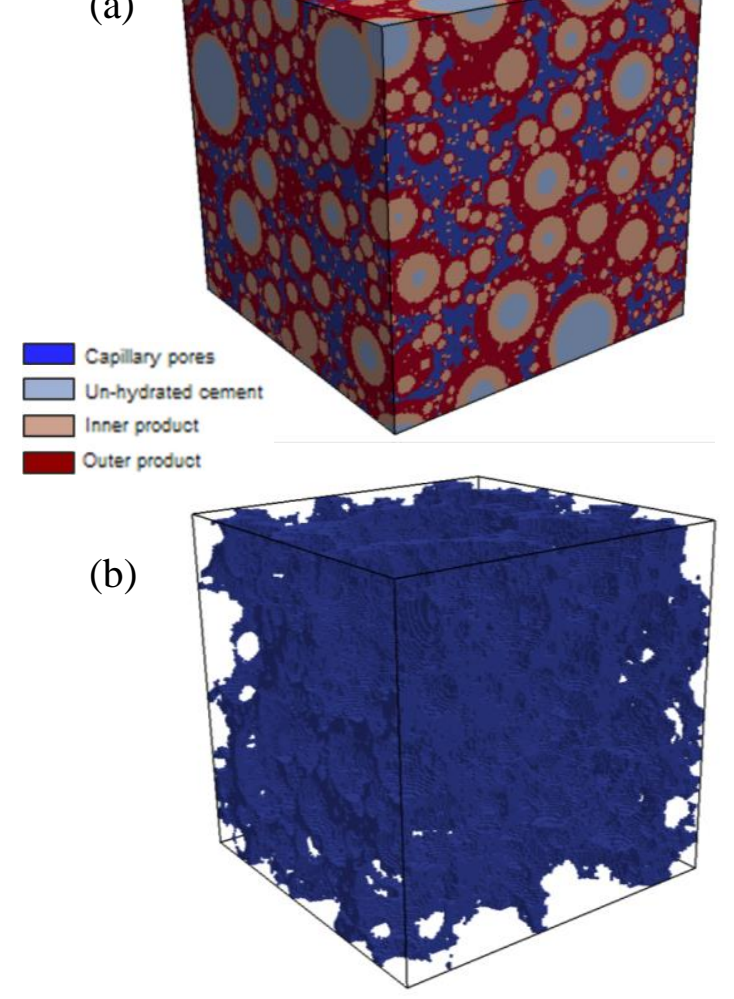

Figure 2: The digitized microstructure and its pore structure of cement paste with w/c $=0.5$ at 26 days.

\section{RESULTS AND DISCUSSION}

\subsection{Fluid flow streamline}

Figure 3 displays the steady-state flow streamline along the flow direction within the corresponding microstructure and pore structure of cement paste. It is clearly seen that the fluid flow is restricted by the solid phase consisting of un-hydrated cement and hydration product, and only passes through the capillary pores. In addition, as expected, some significant changes in the flow velocity can be observed from Figure $3 b$ due to the presence of some pores of small size in the pore network. Since the flow velocity distribution in the pore network is strongly related to the pore size distribution, there exist some positions where the flow velocity is significantly greater than others, as shown in Figure 3b. These positions probably correspond to the pores in small size. These findings further indicate that the LB method provides insight into the internal velocity distribution and allows one to detect preferential flow paths within the specimen, and the LB simulation offers a significant potential for new fundamental insights and understanding of fluid flow processes. 
(a)

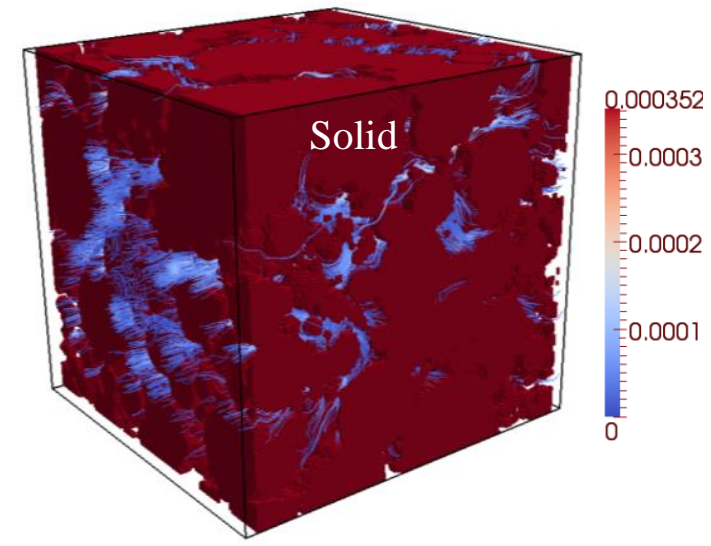

(b)

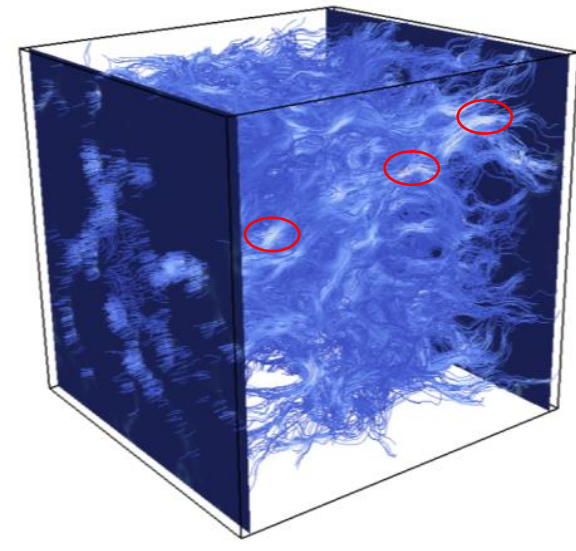

Figure 3: Steady-state flow streamline along the flow direction within the corresponding microstructure and pore structure of cement paste.

\subsection{Permeability}

Following the onset of steady-state flow, the dimensionless intrinsic permeability $\left(\kappa_{l b}\right)$ can be calculated using equation 10 and then converted to the water permeability $\left(k_{w}\right)$ with the dimensions of $\mathrm{m} / \mathrm{s}$. Figure 4 displays the simulated water permeability for cement pastes with w/c ratio of $0.4,0.5$ and 0.6 as a function of degree of hydration. It is obviously that the water permeability decreases with an increase in degree of cement hydration. This is the consequence of the progress of cement hydration. Cement hydration products are gradually formed and fill in some of pores. As a result, the size and the volume of capillary pores decrease and capillary pores start to become disconnected. Accordingly, the water permeability of cement paste reduces as well because the water permeability of cement paste is strongly dependent on the pore size distribution and connectivity of pores. Figure 4 also clearly shows that the samples with higher w/c ratio display higher water permeability. This can be explained by the fact that at the same degree of hydration, more pores are connected with each other in the sample with higher $\mathrm{w} / \mathrm{c}$ ratio.

Figure 5 presents the simulated water permeability for cement pastes with w/c ratio of $0.4,0.5$ and 0.6 as a function of effective porosity. Effective po- rosity is defined as the volume fraction of the connected pores. As expected, the water permeability decreases with a reduction in effective porosity. For the sample with same effective porosity, the permeability values of the samples with different w/c ratios are observed to be very close to each other.

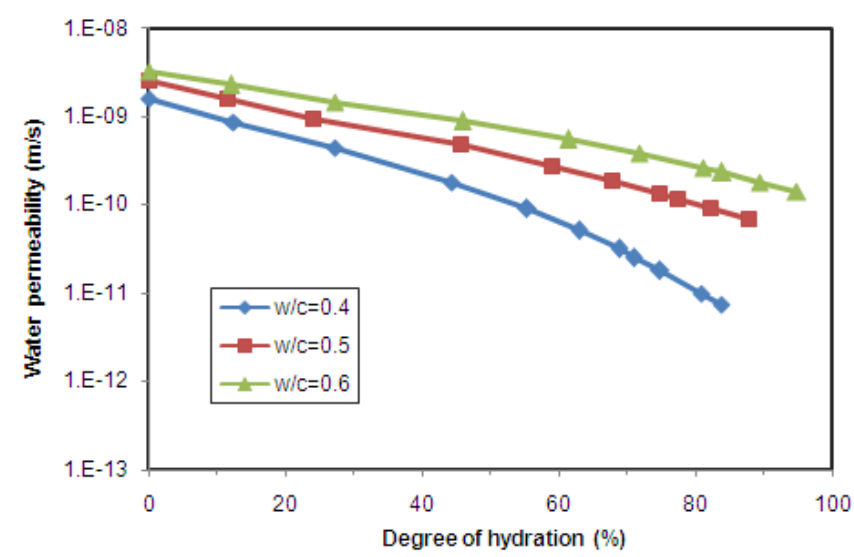

Figure 4: Simulated water permeability as a function of degree of hydration.

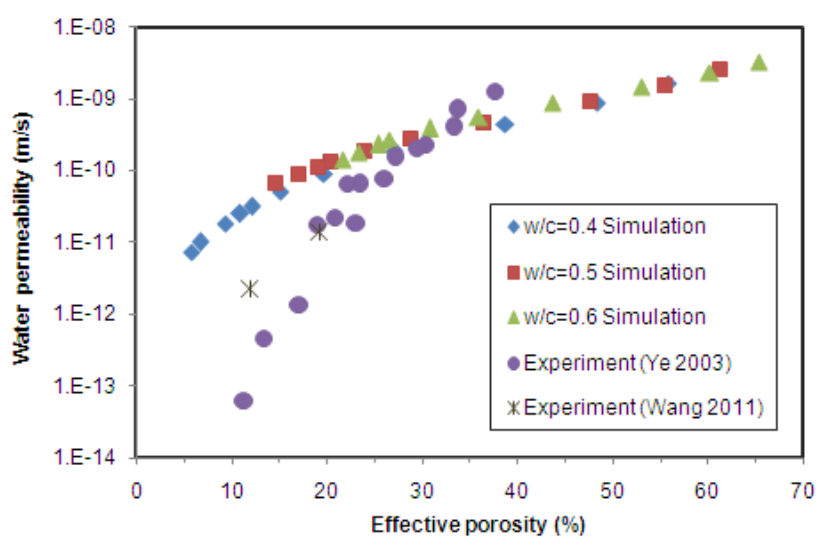

Figure 5: Comparison of simulated and measured permeability as a function of effective porosity.

With the purpose of verification, two sets of experimental data from the literature are selected for comparison. The first set of experimental data is taken from Ye (2003). In his experiment, the used Portland cement is the same as that in the simulation. The w/c ratio was $0.4,0.5$ and 0.6 , respectively. Curing periods of $1,3,7,14$ and 28 days were considered. In order to investigate the relationship between the pore structure and the permeability of cement paste, the mercury intrusion porosimetry (MIP) test was simultaneously performed on the same specimens. The MIP method is able to determine total porosity, ink bottle porosity and effective porosity (Ye 2003). The effective porosity was defined as the total porosity minus the ink-bottle porosity. This part of porosity is considered to form the effective pathway through which the fluid is able to penetrate into the specimen. The second set of experimental data is taken from Wang and Ye (2011). In their experiment, the materials, experimental set-up and proce- 
dures were in accordance with those in the experiment by Ye (2003). The water permeability of cement paste was measured for samples with $\mathrm{w} / \mathrm{c}$ ratio of 0.4 at curing ages of 7, 28 and 90 days. The samples and testing age were the same as the MIP measurements.

A comparison between the simulated and measured permeability as a function of effective porosity is given in Figure 5. It can be found that the simulated permeability shows a good agreement with the measured permeability when the effective porosity is higher than $20 \%$. With a decrease in the effective porosity, the difference between the simulated and measured permeability increases. However, the simulated permeability is still of the same order of magnitude as the measured permeability by Wang and Ye (2011). In addition, for both simulation and experiments, the relationship between the water permeability and the effective porosity is highly correlated. This result implies that the water permeability significantly depends on the effective porosity which involves the information of porosity and connectivity of pores.

\section{CONCLUSIONS}

This work presents a microstructure-based permeability model by using multi-relaxation-time lattice Boltzmann method. From the findings of the present study, the following conclusions can be drawn:

- The LB method provides insight into the internal velocity distribution and allows one to detect preferential flow paths within the specimen, and the LB simulation offers a significant potential for new fundamental insights and understanding of fluid flow processes.

- The simulated water permeability is correlated very well with the microstructure development of cement paste when the degree of cement hydration, w/c ratio and effective porosity are concerned. For the same effective porosity, the permeability values of the samples with different w/c ratios are observed to be very close to each other.

- A good agreement between the simulated and measured permeability can be observed when the effective porosity is higher than $20 \%$. With a decrease in the effective porosity, the difference between the simulated and measured permeability increases. The water permeability significantly depends on the effective porosity which involves the information of porosity and connectivity of pores.

\section{REFERENCES}

Bentz DP. (1997) CEMHYD3D: A three-dimensional cement hydration and microstructure development modelling pack- age, Version 2.0 NISTIR 6485, U.S. Department of Commerce, Washington, DC.

d'Humières D, Ginzburg I, Krafczyk M, Lallemand P, Luo L. (2002) Multiple-relaxation-time lattice Boltzmann models in three dimensions. Philos T Roy Soc A 360(1792):437451.

Lallemand P, Luo L. (2003) Theory of the lattice Boltzmann method: Acoustic and thermal properties in two and three dimensions. Phys Rev E 68036706.

Marchand J, Gerard B. (1997) Microstructure-based models for predicting transport properties, In Reinhardt H.W. (eds), RILEM Report 16: Penetration and Permeability of Concrete: $41-81$.

Pan C, Hilpert M, Miller CT. (2004) Lattice-Boltzmann simulation of two-phase flow in porous media. Water Resour Res $40 \mathrm{~W} 01501$.

Qian Y, d'Humières D, Lallemand P. (1992) Lattice BGK models for Navier-stokes equation. Europhys Lett (17):479-484.

van Breugel K. (1991) Simulation of hydration and formation of structure in hardening cement-based materials. Ph.D. Thesis, Delft University and Technology, Delft, The Netherlands.

Wang Y, Ye G. (2011) Effect of micronized sands on the water permeability of cementitious material. In Ángel Palomo, Aniceto Zaragoza, Juan.Carlos López Agüí (eds.), The proceeding of 13th International Congress on the Chemistry of Cement. Madrid, Spain: Los Autores: 1-7.

Ye G. (2003) Experimental study \& numerical simulation of the development of the microstructure and permeability of cementitious materials. Ph.D. Thesis, Delft University and Technology, Delft, The Netherlands.

Zhang M, Ye G, van Breugel K. (2011) Lattice Boltzmann simulation of the ionic diffusivity in cement paste. In Leung C, Wan KT (eds.), Proc. Int. RILEM Con. on Advances in Construction Materials Through Science and Engineering, RILEM Publications Sarl, Bagneux, France: 469-477.

Zou Q, He X. (1997) On pressure and velocity boundary conditions for the lattice Boltzmann BGK model. Phys Fluids 9(6):1591-1598. 